\title{
Open reduction and percutaneous pinning via lateral approach without using C-arm in Gartland type III supracondylar humerus fractures in children
}

\author{
Authors \\ Tanveer Ahmed Bhat', Malik Naseer Ahmad ${ }^{2}$, Imtiyaz Ahmad Beigh ${ }^{3 *}$, \\ Mohammad Farooq Butt ${ }^{4}$, Zubair Ahmad lone ${ }^{5}$, Tanveer Ali ${ }^{6}$, Karanbir Singh ${ }^{7}$ \\ ${ }^{1,3}$ Senior Resident, Orthopaedics, SGGRIM \& HS, Dehradun \\ ${ }^{2}$ Senior Resident, Government Medical College, Baramulla, J\&K \\ ${ }^{4}$ Assistant Professor, Orthopaedics, GMC, Jammu \\ ${ }^{5,6,7}$ PG Y3, Department of Orthopaedics, Govt. Medical College Jammu \\ *Corresponding Author \\ Imtiyaz Ahmad Beigh
}

\begin{abstract}
Introduction: The outcomes of open reduction of displaced supracondylar humerus fractures in children via lateral approach with percutaneous pinning without using $C$-arm were evaluated.

Methods: Thirty six consecutive patients with Gartland type III fractures were consecutively treated between June 2016 to January 2019 in our hospital. The mean follow-up period was 12 months (6-15 months). The patients were analysed according to the Flynn criteria.

Results: After an average follow-up of 12 months, the results were excellent in 26, good in 4, fair in 3, and poor in 3. Only 2 cases needed revision surgery due to unacceptable reduction. There was one iatrogenic ulnar nerve injury and two cases of pin tract infection which resolved with antibiotics.

Conclusion: Open reduction and percutaneous pinning is a safe and effective procedure to treat supracondylar humerus fractures in children and can be done without $C$-arm with fairly good accuracy.

Keywords: percutaneous pinning; fluoroscopy; supracondylar; closed reduction; open reduction.
\end{abstract}

\section{Introduction}

Supracondylar humerus fractures $(\mathrm{SCH})$ are the most common fractures about the elbow in children and have a peak incidence at the age of 5 to 6 years ${ }^{[1]}$. Type I and II are usually treated nonoperatively. Type III fractures accounting for approximately $97 \%$ to $99 \%$ of SCH fractures are usually caused by a fall onto the outstretched hand with the elbow in full extension ${ }^{[2]}$. Soft tissue and neurovascular injuries often accompany these fractures. These have extension in the sagittal plane and rotation in the frontal and/or transverse planes. Closed reduction and percutaneous pinning (CRPP) using intra-operative fluoroscopy is the preferred treatment strategy. In our set-up as C-arm imaging was not available in emergency operation theatre we operated all the cases included in our study group by open reduction percutaneous pinning (ORPP). This protocol not only avoided delayed treatment in busy main 
operation theatre but also decreased complications related to fracture deformity and elbow swelling as well as alleviated the parental anxiety.

ORPP of SCH fractures can be performed by a lateral, medial, anterior, or posterior approach ${ }^{[3]}$. We chose a lateral approach as this was easy, required minimal dissection, provided good access to fracture site and avoided iatrogenic damage to neurovascular structures. The purpose of this study was to evaluate the outcomes of $\mathrm{SCH}$ humerus fractures treated by ORPP via lateral approach without using $\mathrm{C}$-arm imaging.

\section{Patients and Methods}

A total of 44 patients with type III SCH fractures presented to the emergency room in our institution between June 2016 and January 2019. Three patients had skin blisters and two had compartment syndrome developed due to mismanagement by traditional bonesetters. One patient presented with pulseless cool hand and had brachial artery injury. Vascular repair together with fracture reduction was done via anterior approach. 2 cases had unacceptable reductions on post-operative radiographs and were re-operated in our main operation theatre under C- arm. All these 8 cases were excluded from the study.

In this retrospective study we included 36 patients which were consecutively treated in our emergency operation theatre by ORPP via lateral approach. The patients consisted of 28 boys and 8 girls with a mean age of 7.5 years (range 3-14 years). Four cases in the study group had pink pulseless hand and were urgently fixed and observed for an average of five days postoperatively. Two cases were open type I fractures and were also included in the study. Two patients had sustained ipsilateral femoral shaft fracture and were managed by intramedullary elastic nails. One patient had ipsilateral distal radius fracture which was managed nonoperatively.

Baumann's angle and the relationship of the capitellum to the anterior humeral line (Fig. 1) were used for determining the adequacy of reduction $^{[4]}$. Flynn $^{[5]}$ grading system was used to assess the results of treatment (Table 1).

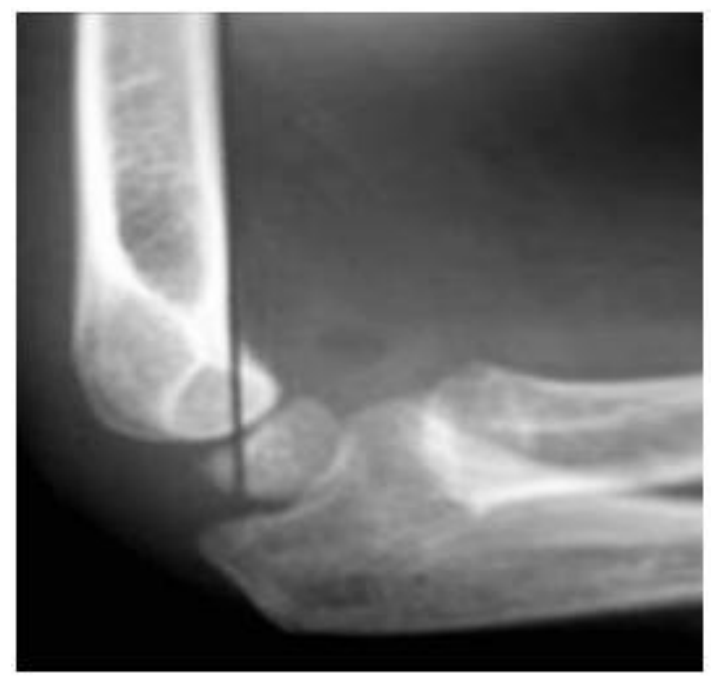

(a)

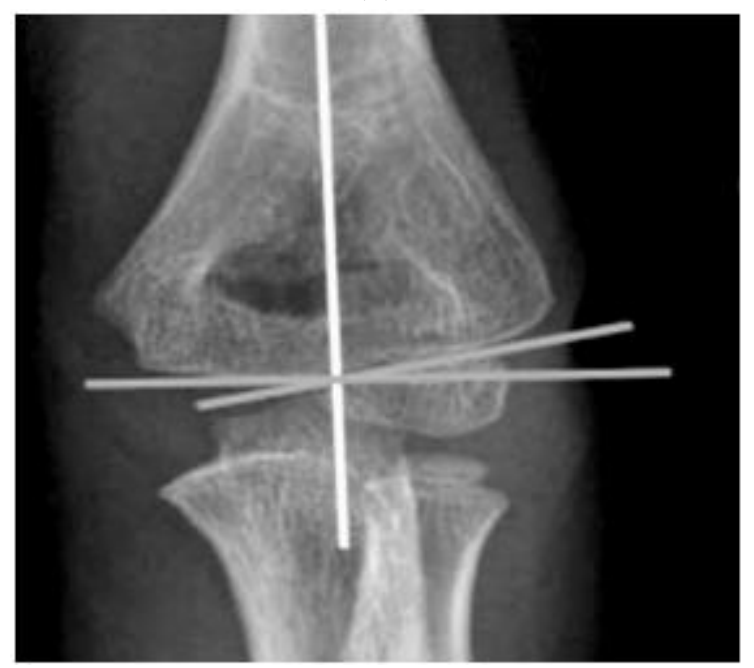

(b)

Fig. 1 Drawing anterior humeral line (a) transecting capitellum and Baumann angle (b) on radiographs

Table 1 Flynn Criteria

\begin{tabular}{|l|c|c|}
\hline Result & $\begin{array}{c}\text { Loss of Baumann } \\
\text { angle (degrees) }\end{array}$ & $\begin{array}{c}\text { Loss of motion } \\
\text { (degrees) }\end{array}$ \\
\hline Excellent & $0-5$ & $0-5$ \\
\hline Good & $6-10$ & $6-10$ \\
\hline Fair & $11-15$ & $11-15$ \\
\hline Poor & $>15$ & $>15$ \\
\hline
\end{tabular}

\section{Surgical Technique}

After general anaesthesia, patients were placed in a lateral decubitus position. Pneumatic tourniquet was used in all cases. Open reduction was performed by a $3-5 \mathrm{~cm}$ curvilinear skin incision over the lateral aspect of elbow. After blunt 


\section{JMSCR Vol||07||Issue||11||Page 640-645||November}

dissection, fracture was visualised by using small Hohmann elevators and any interposing structure was removed. Fracture hematoma was curetted and wound irrigation was done. Reduction was achieved by applying pressure by a thumb on the proximal fragment and traction performed on the forearm while flexing the elbow. Reduction was maintained by manual pressure of the assistant. After reduction, lateral or crossed smooth K-wires (1.5 or $1.8 \mathrm{~mm}$ ) were inserted percutaneously (Figures 2 and 3). To insert the K-wire from the medial side care was taken to protect ulnar nerve. For this elbow was extended and the epitrochlea was palpated with thumb and then the thumb was moved posteriorly. In some cases with gross swelling ulnar nerve was protected by using a mini- incision. Various pin configurations were used (Table 2).

Table 2Pin configurations used for surgical fixation

\begin{tabular}{|l|c|}
\hline Pin configuration & Number of cases \\
\hline 3 lateral pins & 4 \\
$\quad$-all parallel & 3 \\
-divergent & \\
\hline 2 lateral pins & 4 \\
-both parallel & 1 \\
$\quad$-divergent & 11 \\
\hline 2 lateral pins and one medial pin & 13 \\
-parallel lateral pins & \\
-divergent lateral pins & \\
\hline
\end{tabular}

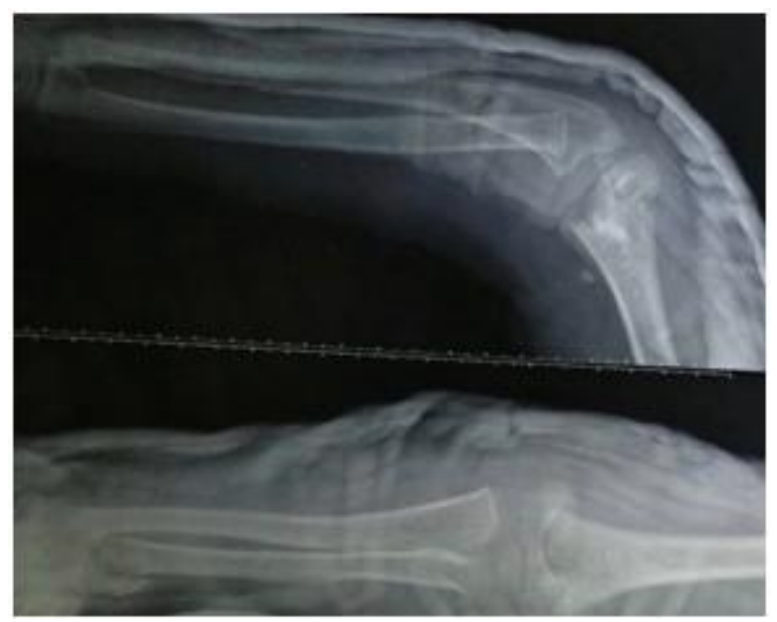

(a)

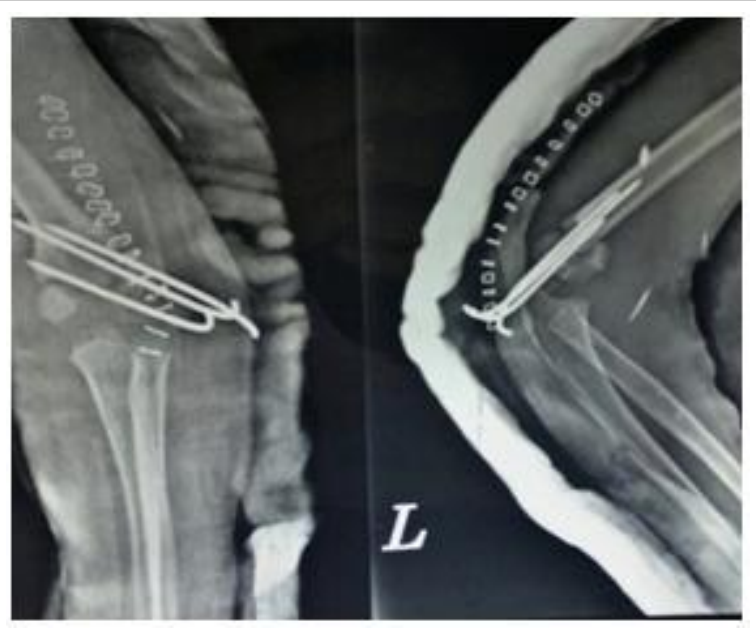

(b)

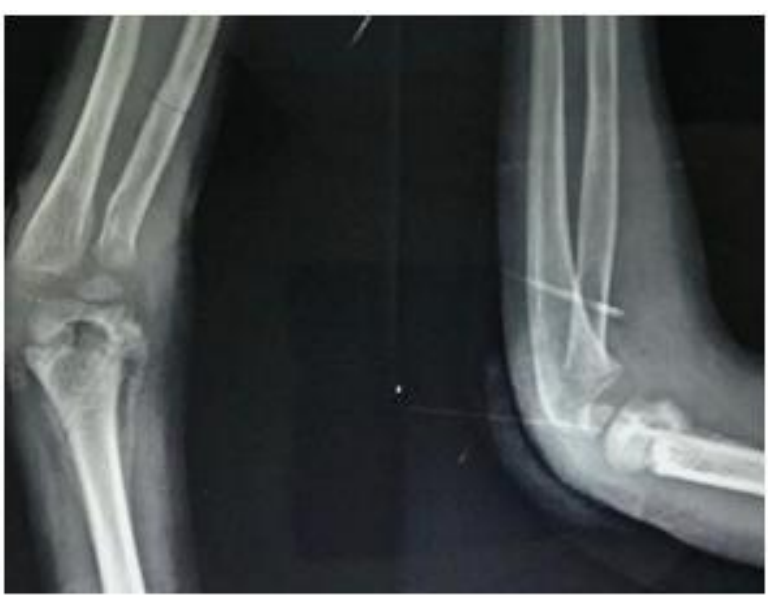

(c)

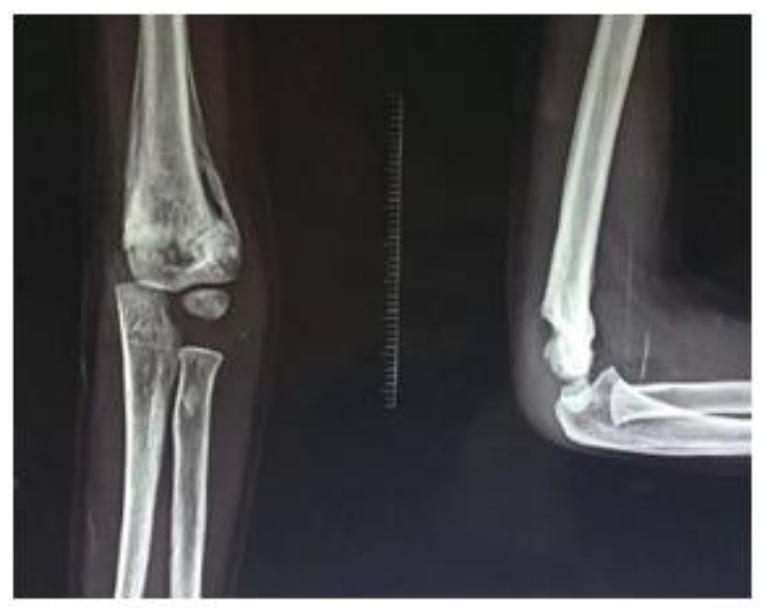

(d)

Fig. 2A case of type III SCH fracture in a 6y old boy (a),fixed by ORPP with 3 lateral parallel Kwires(b), at 3 weeks after removing K-wires(c), at 4 month follow-up (d). Distal radius fracture in this case was managed conservatively 


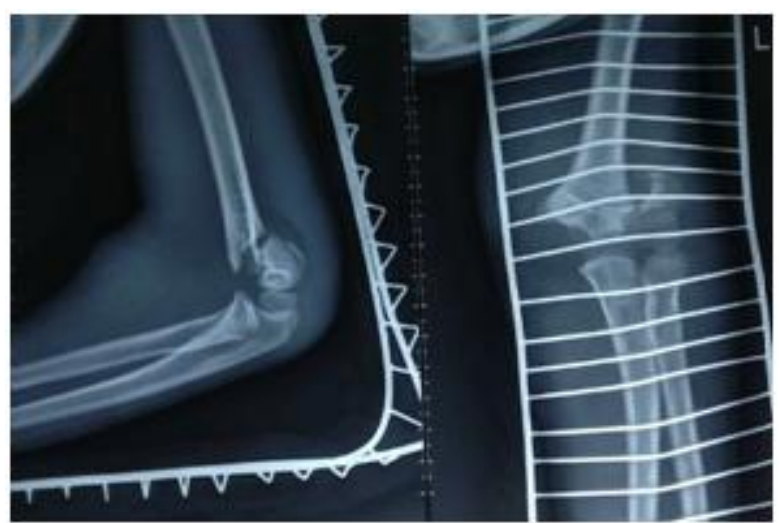

(a)

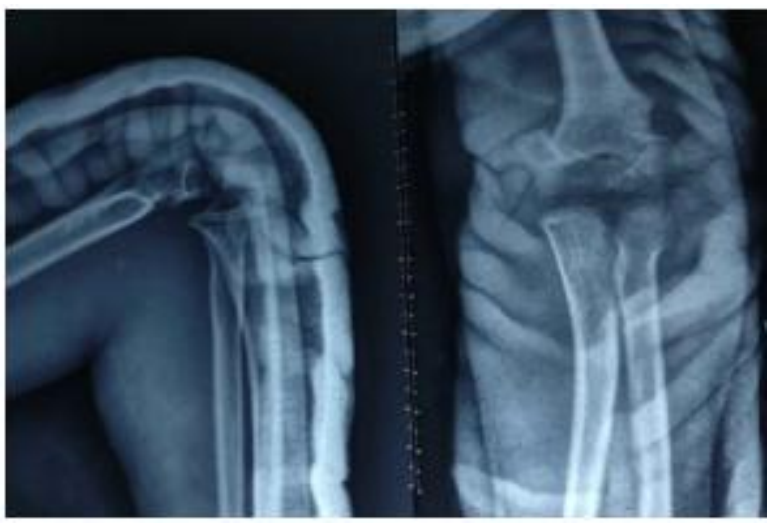

(b)

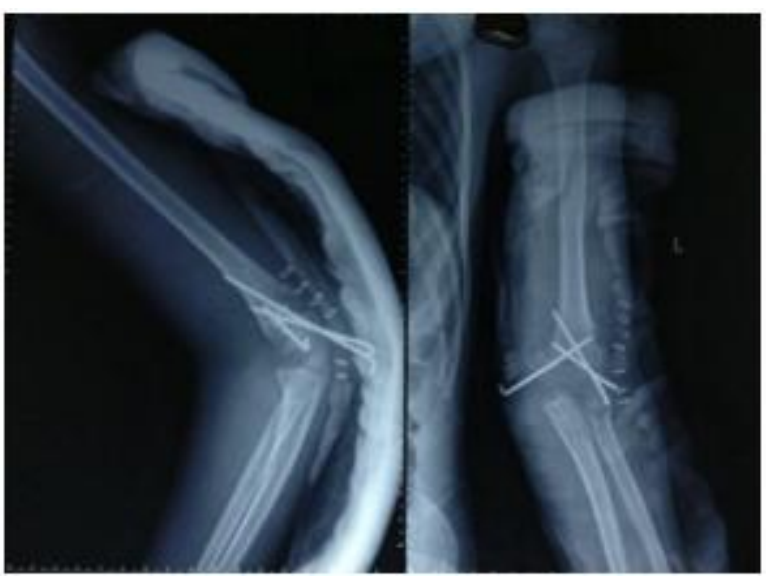

(c)

Fig. 3A case of extension type $\mathrm{SCH}$ fracture in a 10 y old boy (a), initially reduced in a POP slab (b), fixed by using 2 divergent K-wires laterally and $1 \mathrm{~K}$ - wire from medial side (c), ulnar nerve was protected in this case by using a mini-incision

As the C-arm was not available in our emergency operating theatre confirmation of the reduction was performed with direct radiography. 2 patients had unacceptable reduction on post-operative radiographs and needed re-operation. These were excluded from the study group. A long arm POP slab was applied in 50 - 70 degrees of flexion depending on pre-operative swelling. The patients were discharged $12-24 \mathrm{~h}$ after surgery. The pins and POP slab were removed in the OPD after 3 weeks followed by active assisted range of motion (ROM) exercises of the elbow.

\section{Results}

Pre-operatively, there were $4(11.11 \%)$ anterior interosseous nerve (AIN) injuries, with the typical presentation of pointing index finger. All cases resolved spontaneously within 6 weeks after injury. The average duration $\mathrm{f}$. presentation in the emergency to the surgery was 8 hours (range, 415 hours). Average duration of surgery was 45 minutes (range, 37- 55 minutes). Average followup was 12 months (range, 6-15 months). Average time to union was 6.5 weeks (range 6 to 8 weeks). At the final follow-up, the mean difference in Baumann angle was $4.5^{\circ}$. One patient had a Baumann angle difference of more than $15^{\circ}$. Three patients had more than $15^{\circ}$ difference in ROM compared to that of the non-injured extremity. 26 patients had excellent results, 4 had good results, 3 had fair results, and 3 had poor results (Table 3 ). The results were much better in terms of Baumann angle difference than in terms of average ROM achieved. In the patients with poor functional results, stiffness, and loss of motion were marked and needed prolonged physiotherapy. ORPP without using image intensifier not only had good results in terms of clinico- radiological healing but also avoided exposure of radiation and was time saving.

Table 3. Final Results

\begin{tabular}{|l|c|}
\hline Result according to Flynn criteria & Number of patients \\
\hline Excellent & 26 \\
\hline Good & 4 \\
\hline Fair & 3 \\
\hline Poor & 3 \\
\hline
\end{tabular}

\section{Complications}

Two (5.55\%) patients had a superficial pin track infection that resolved with oral antibiotics and dressings. Additionally, there was one (2.7\%) 
iatrogenic ulnar nerve injury which resolved completely by the fourth postoperative month. One patient developed myositis ossificans around the elbow. 2 patients needed revision surgery under $\mathrm{C}$-arm as the reduction was not acceptable on post-operative radiographs. These two cases were excluded from the study.

\section{Discussion}

CRPP under C-arm imaging is a standard surgical treatment for displaced $\mathrm{SCH}$ fractures ${ }^{[6]}$. In our set-up C-arm imaging was not available in emergency operation theatre. This coupled with the fact that oftentimes intraoperative closed reduction attempts do not yield satisfactory alignment of the fracture, we managed all our cases by ORPP. The purpose of this study was to determine whether ORPP via lateral approach of displaced $\mathrm{SCH}$ fractures without using $\mathrm{C}$-arm is safe and effective procedure or not.

Although concerns about ORPP is iatrogenic softtissue injury, we did meticulous dissection and fracture reduction in our patients and didn't encounter any major complications except one ulnar nerve injury during pin insertion. Bo Gou et al. in a meta-analysis found no significant differences between the two methods (CRPP and ORPP) for the results of carrying angle, Bauman angle, and complication rate ${ }^{[7]}$. In this study, four patients $(11.11 \%)$ had pre-operative neurological (AIN) injury. Babal et al. in a meta-analysis on nerve injuries associated with pediatric $\mathrm{SCH}$ fractures reported injury rate of AIN in the range of $0-21 \%{ }^{[8]}$. We encountered iatrogenic nerve injury rate of $2.7 \%$ which is similar to that reported in literature ${ }^{[9]}$. Loss of reduction was seen in 2 patients on post-operative radiographs and needed re-operation under fluoroscopic guidance. These were type IV SCH fractures with multidirectional instability and couldn't be diagnosed during the first procedure as they are usuallydetermined on fluoroscopy when on a lateral view the capitellum is anterior to the AHL with elbow flexion, and posterior to the AHL with elbow extension ${ }^{[10]}$. Superficial pin tract infections range from 2 to $7 \%$ in the literature ${ }^{[11]}$. The rate in our cases was $5.5 \%$. We had good functional results with lateral approach. Keskin and Sen concluded that in ORPP practices, lateral incision is a simple and reliable approach despite a dissatisfying scar tissue formation ${ }^{[12]}$

The limitation of this study is that not all the cases operated were similar. Though all were classified as type III injuries, some cases were easy to reduce and had stable fixation. Some cases had brachialis muscle, brachial artery and median nerve interposition and were difficult to reduce. Additionally there were two open fractures which needed wound irrigation and debridement. This also resulted in increase in the operative time in some cases. Another limitation of our study is that the cases were performed by more than one surgeon.

\section{Conclusion}

ORPP is a safe and effective procedure to treat $\mathrm{SCH}$ fractures and can be done without $\mathrm{C}$-arm with fairly good accuracy. This helps in early fracture stabilisation, prevents multiple manipulations that are required in closed methods and is indispensable in treating those injuries with associated brachialis muscle or neurovascular structure interposition between fracture fragments.

\section{References}

1. Minkowitz B, Busch MT. Supracondylar humerus fractures. Current trends and controversies. OrthopClin North Am. 1994; 25:581-594.

2. Mahan ST, May CD, Kocher MS. Operative management of displaced flexion supracondylar humerus fractures in children . J Pediatr Orthop. 2007 ; 27(5):551-556.

3. Nuri AC, Ali T, Muhtar OA, Murat A, Bulent $\mathrm{O}$, Yalcin TA. Open reduction via posterior triceps sparing approach in comparison with closed treatment of posteromedial displaced Gartland type III supracondylar humerus fractures . Journal of Pediatric Orthopaedics B. 2008 ; 17(4): 171-178. 
4. Otsuka NY, Kasser JR. Supracondylar fractures of the humerus in children. $J A m$ Acad Orthop Surg. 1997; 5(1):19-26.

5. Flynn JC, Matthews JG, Benoit RL. Blind pinning of displaced supracondylar fractures of the humerus in children. Sixteen years experience with long-term follow-up . J Bone Joint Surg Am. 1994 ; 56:263-272.

6. Davis RT, Gorczyca JT, Pugh K. Supracondylar humerus fractures in children: Comparison of operative treatment methods . ClinOrthop. 2000 ; 376: 49-55.

7. Gou B, Wang X-T, Zhang Q-S, Wang Q-B. Open or closed reduction and percutaneous pinning for pediatric displaced supracondylar humerus fractures: a meta-analysis and system review. Int J ClinExp Med. 2018; 11(10):10278.10286.

8. Babal JC, Mehlman CT, and Klein G. Nerve injuries associated with pediatric supracondylar humeral fractures: A Metaanalysis. J Pediatr Orthop. 2010 ; 30:253263.

9. Skaggs DL, Hale JM, Bassett J, Kaminsky C, Kay RM, Tolo VT. Operative treatment of supracondylar fractures of the humerus in children. The consequences of pin placement. J Bone Joint SurgAm. 2001 ; 83:735-740.

10. Skaggs DL and Flynn JM. Supracondylar fractures of the distal humerus. In: Flynn JM, Skaggs DL, Waters PM, eds.Rockwood \& Wilkins' Fractures in Children. Philadelphia, Wolters Kluwer, 8th edn, 2015: pp 590.

11. Mehlman CT, Strub WM, Roy DR, Wall EJ, Crawford AH. The effect of surgical timing on the perioperative complications of treatment of supracondylar humeral fractures in children. J Bone Joint SurgAm. 2001 ; 83:323-327.
12. Keskin D, Sen H. The comparative evaluation of treatment outcomes in pediatric displaced supracondylar humerus fractures managed with either open or closed reduction and percutaneous pinning. Acta Chirurgiae Orthopaedicae Et Traumalogiaecechosl. 2014 ; 81, 380-386. 